\title{
TOWARDS A CONCEPT FOR A HIDDEN OBJECT GAME WITH DYNAMIC DIFFICULTY ADJUSTMENT
}

\author{
Daniel Atorf ${ }^{1}$, Sergius Dyck ${ }^{1}$ and Jonas Steinbach ${ }^{2}$ \\ ${ }^{1}$ Fraunhofer IOSB, Fraunhoferstr. 1, 76131 Karlsruhe, Germany \\ ${ }^{2}$ Hochschule Worms, Erenburgerstraße 19, 67549 Worms, Germany
}

\begin{abstract}
This short paper elaborates on a work in progress concept for a hidden object game in the knowledge domain of remote sensing. Previous evaluations of the serious game Lost Earth 2307 revealed the need for shorter gameplay variants while still supporting the main learning objectives. It also affirmed the need to individually improve player immersion. To address this need, the short paper briefly depicts the method of the adaptivity cycle by Shute and Zapata and dynamic difficulty adjustment (DDA). The DDA is a popular method to adapt games and requires the finding of relevant difficulty levels and applying it in the game. The process of mapping and applying the difficulty in the game is called parametrization. This short paper shows a first concept of how to parameterize an adaptive game by a prototype implementation of an adaptive hidden object game. The short paper concludes with the results of a first informal test of the implementation and further steps.
\end{abstract}

\section{KEYWORDS}

Digital Game-Based Learning, Adaptive Game Design, Immersion, Flow

\section{INTRODUCTION}

The basic idea for this concept derived from the serious game Lost Earth 2307. It was developed for teaching on how to analyze aerial and satellite images and on how to formally describe the identified objects by using specific terms and write a report. This training domain is rather complex to teach, therefore individual training courses last several months and trainers have a high interest in keeping the students motivated over a long time. The game addressed this need by following the ideas of Digital Game Based learning (Prensky 2007) and Immersive Didactics (Bopp 2006). It was integrated into the course as an optional element in the informal learning phase where students had to intensify their knowledge through exercises.

Iterative evaluations during development and further on-site evaluations showed a need for streamlining the game design (Atorf et al. 2020). A main issue was the overall needed time to complete a mission. The initial type of task covered actions on each step of the image exploitation process cycle. This mapped exactly the exercises given to students for the informal learning phase in the afternoons. However, this kind of task required several hours to complete, thus there was not enough time for students to play "just another mission" in addition to the regular exercises. Therefore, missions in the game needed to evolve into smaller and more accessible tasks while keeping the major learning goals. In addition, there was a need to individually improve player immersion.

This short paper describes a work in progress concept and implementation for a hidden object game. This game genre promises to enable tasks which are quite close to actual image interpretation tasks, hence supporting original learning goals but providing a smaller and more fun task. The paper also describes the concept behind the parameterization of the game in order to enable a dynamic difficulty adjustment (DDA). The DDA addresses the individual improvement of player immersion. Finally, a first informal test of the concept is depicted and further steps are discussed. 


\section{METHOD}

Analog to the original game Lost Earth 2307, the concept still follows the ideas of Digital Game Based Learning by Prensky and Immersive Didactics by Bopp. This means the game concept should support immersion or as Prensky states "[...] the top two reasons people say they play interactive games [...] is because they are challenging and relaxing. This formulation seems very close to that magical state of motivation some refer to as flow." Immersion and flow act as a vehicle for facilitating positive user experience in order to maximize the impact of educational games (Kiili 2005).

Cairns et al define immersion in games as "the engagement or involvement a person feels as a result of playing a digital game" (Cairns et al. 2014). They describe three levels of immersion: engagement (player invests time and effort to play a game), engrossment (player pays a lot of attention to the game and is also emotionally involved) and total immersion (player "feels in the game"). According to Csikszentmihalyi, flow describes an "automatic, effortless, yet highly focused state of consciousness" (Csikszentmihalyi 1997). Cairns' Total Immersion can be compared to the state of flow (Kannegieser 2019). To sum up, a good serious game must enable player immersion and ideally create a state of flow.

To keep the user constantly in a state of immersion, thus constantly in the state of flow, the game must support some sort of adaptivity. Dynamic difficulty adjustment (DDA) is a possibility to keep the balance between challenge and skill (see Figure 1, left). The model of Shute \& Zapata (see Figure 1, right) is suitable for the design of an adaptive system. The system monitors the user in a cycle and adapts itself to his needs (Shute and Zapata 2012). This adaptive cycle consists of four phases. In "Capture," a learner interacts with the system and all relevant data from the user interaction (e.g., scores, errors, engagement, etc.) are captured. In "Analyze", the data from the interaction is analyzed and fed to a learner model. The learner model describes the learner's knowledge of the respective learning domain. In "Select", new information and learning strategies relevant for the learner are selected based on the learner model, learning domain and learning goals. In "Present" the selected information is visualized and presented to the learner.
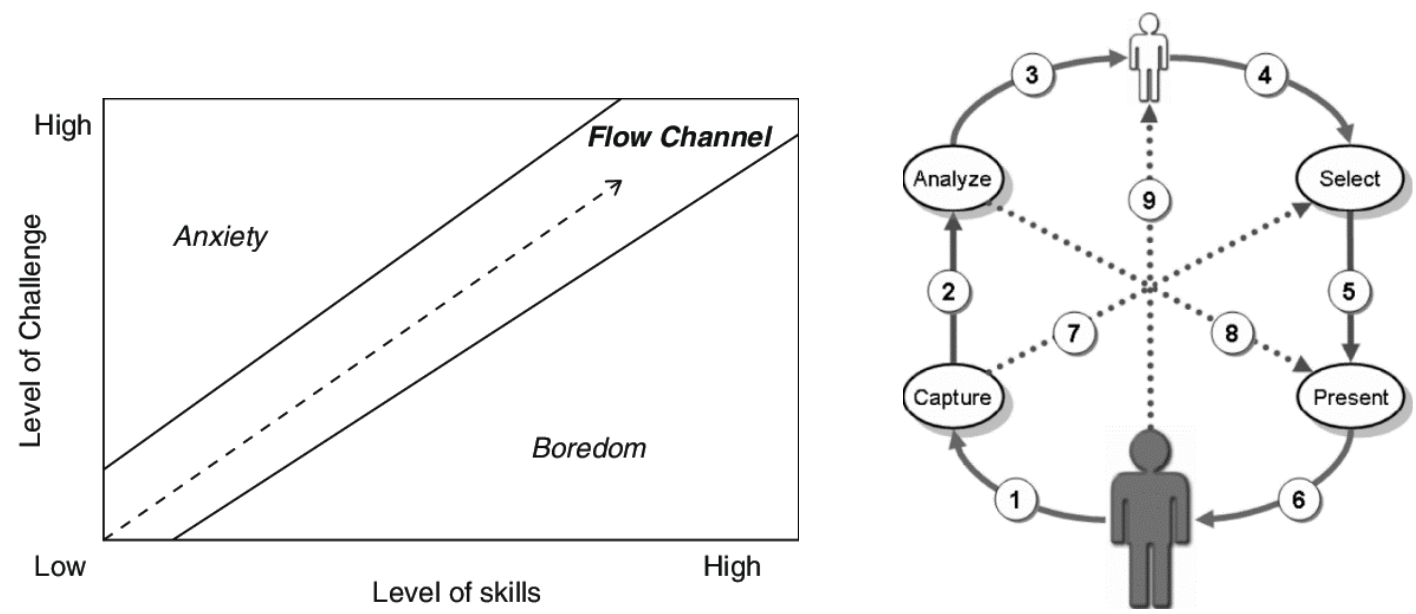

Figure 1. Left: Flow channel (Csikszentmihalyi 1997); Right: Adaptivity cycle (Shute \& Zapata 2012)

For the concept of the game "Present" is especially relevant, as the game, which the user sees and plays, acts as the presenter. Hence, the game concept assumes a successful loop through the cycle and a corresponding response from the "Select" phase, the so-called Adaptivity Response (AR). The AR expresses the difficulty in a few scalar, normative parameters (Streicher 2020): Performance (current performance of the user), Assistance (whether and when the user should get help), and Skill (long-term performance of the user, i.e., how well the user has performed in e.g., exams). As said, "Present" includes the nature and structure of the game in relation to the learner model. For this concept, this includes a dynamic adaptation of difficulty (DDA) to the user, and accordingly, enabling parameterization of the game. The parametrization answers the question of "what to adapt", i.e. how the game has to be structured so that it can be adapted. 


\section{CONCEPT AND IMPLEMENTATION}

The general game idea is based on the concept of a "Wimmelbilderbuch" or hidden objects. A classic example of hidden objects is the book series "Where's Wally?"(Handfort 1987) in which the main character named Wally has to be found on every page. In the field of computer games, there is the commercially successful genre of "hidden object games". Hidden object games are immersive and tell a story (Ioannidou 2018). Often the player is assigned to solve a given task. To do this, he has to move from one scene to the next and find various "hidden" objects within them. The essence of hidden object games is similar to the learning goal "Typenkunde" in image exploitation. An image analyst must not only recognize whether and where a relevant object can be seen on an image, but also what kind of object it actually is. This makes this type of interaction almost identical to that in hidden object games. And due to its immersive nature, they also meet requirements of DGBL.

The implemented game world for the hidden object game is based on a generated map. The current map section can be repositioned and zoomed in and out. The map shows vehicles to be found (target objects) and other objects (distractors) that resemble the target objects and/or are intended to distract the player. The player has to mark the target objects in a given time. Correctly marked objects are awarded points, incorrectly marked objects result in a penalty. The speed with which the objects were correctly marked has a positive effect on the total score. Figure 2 shows a screenshot of the game.

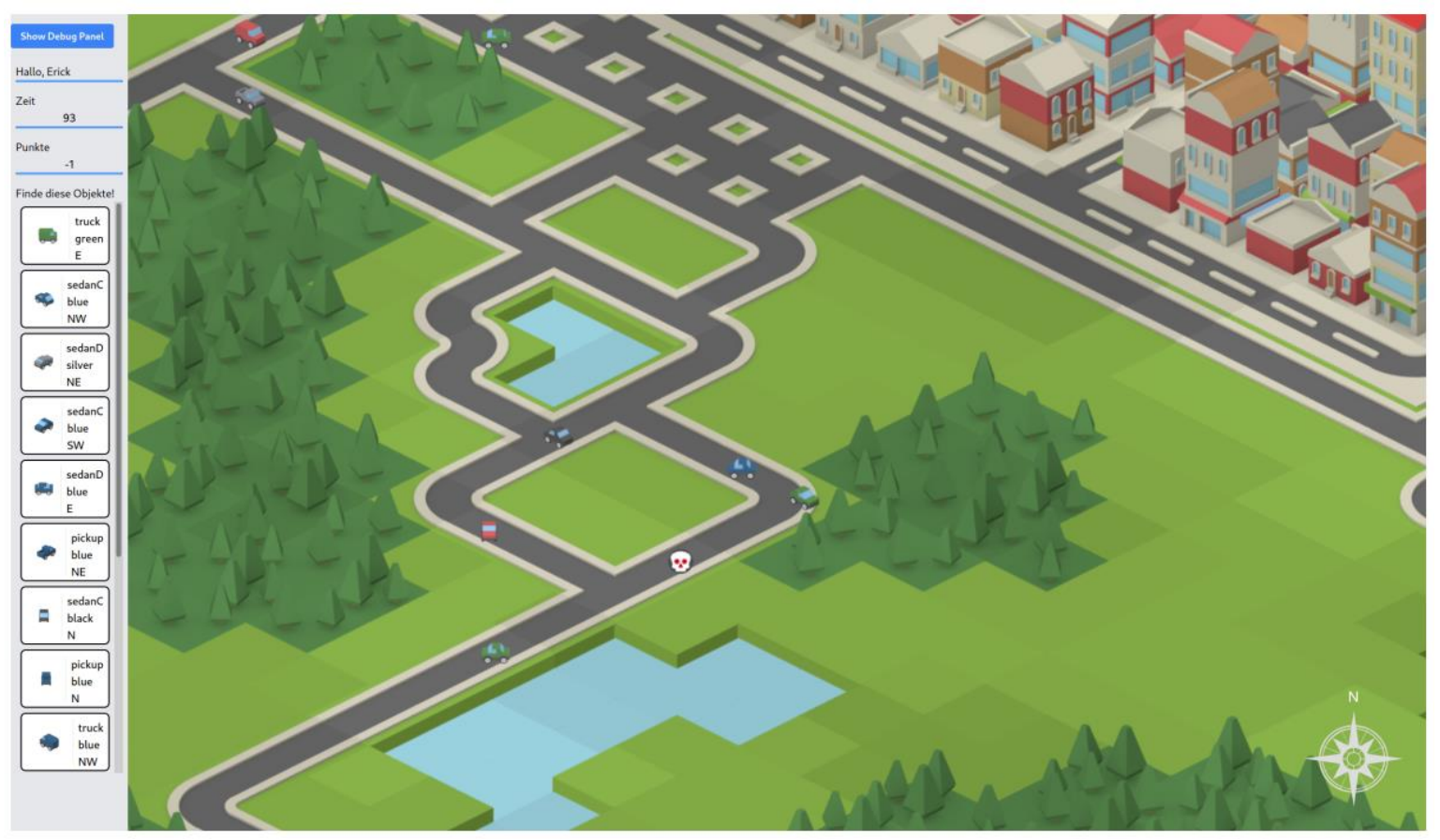

Figure 2. screenshot Hidden Object game (Left: list of objects, score, remaining time; Right: Scene)

With each new game, a new map is generated and the target objects as well as the distractors are repositioned. In addition to pure placement, options for customizing the target objects and distractors are also provided to enable training of "Typenkunde". Hence, the target objects are vehicles differing in shape, size, color and orientation, representing the characteristics mentioned by Lillesand (Lillesand et al 2015). The scene is procedurally generated using the tiling method and Perlin Noise to generate the landscape in the scene (Perlin 1985). During the game, other perturbations are added (e.g. day\&night cycle). An adjustment of the difficulty level according to DDA is achieved by changing the map size, type of vehicles, number of vehicles to be found, number of distractors and the time available. To be more precise, there is a mapping on the adaptive response message parameters. The performance parameter controls the generation of the map, especially the size of the map and proportions of streets and cities. The assistance parameter controls the level of help and was realized in two variants: Highlighting of occasional target objects on the map (1) and displaying a thumbnail of the sought target objects in the list of objects to be found (2). The skill parameter controls target objects and distractors. It determines the number of different vehicle variants, the distribution of vehicles on the map, the number of vehicles and distractors, the game duration and the day\&night cycle. 


\section{DISCUSSION \& FURTHER STEPS}

There is no universal guide for creating adaptive games, but the ideas of DGBL were applied by implementing a hidden object game. Following the model of Shute \& Zapata the game adapts in the "Present" phase by implementing the adaptive response parameters, enabling DDA.

Five participants (3 image interpreters) went through a very first test of the game. A test followed two phases. In phase 1 participants played two complete rounds of the game as a 'Think Aloud Session', where participants were asked to verbalize what happens inside their head (Albert and Tullis 2013). The second game round in this phase was used to adjust the difficulty. Phase 2 was interviewing the respective participant. According to the think aloud sessions and the interviews, the vehicles used in the game are suitable for the application context. But the sprites used are too small and need to be improved (see Lillesand et al. 2015). The test users especially liked the number of distractor variants, describing them as "creative" and "promising". The concept showed that there is a great potential for creativity here, and that a great variety of distractors is possible. The use of time as an adaptable dimension to create stressful situations, seemed to be a promising approach and should be further explored in the next steps.

Overall the concept and the first prototype implementation seems promising. Further steps will include a formal evaluation with the target audience, especially focusing on fun and on learning goals.

\section{REFERENCES}

Albert, W., Tullis, T., 2013. Measuring the user experience: collecting, analyzing, and presenting usability metrics. Morgan Kaufmann, Waltham.

Atorf, A. et al. 2020. Towards A concept for streamlining game design of an existing serious game and preliminary evaluation. In Proceedings of the IADIS International Conference WWW/Internet 2020, pp. 155 - 160.

Bopp, M. 2006. Immersive Didaktik und Framingprozesse in Computerspielen. In Neitzel, B. and Nohr, R.F. Das Spiel mit dem Medium. Partizipation-Immersion-Interaktion. Zur Teilhabe an den Medien von Kunst bis Computerspiel, pp. 170-186, Schüren, Marburg.

Cairns, P. et al. 2014. Immersion in Digital Games: Review of Gaming Experience Research. In Handbook of digital games 1, p. 767.

Csikszentmihalyi, M. 1997. Flow and the Psychology of Discovery and Invention. In Harper Perennial, New York, p 39.

Handfort, M. 1987. Where's Wally? Walker Books.

Ioannidou. E. 2018. Neo-Victorian Hidden Object Games, Participatory Culture, and the Interaction Between Past and Present. In Adaptation 11.2, pp. 159-170.

Kannegieser, E. et al. 2019. Conducting an Experiment for Validating the Combined Model of Immersion and Flow. In Proceedings of the 11th International Conference on Computer Supported Education, Vol 2, pp. 252-259.

Kiili, K. 2005. Digital Game-Based Learning: Towards an Experiential Gaming Model. In The Internet and Higher Education 8.1. pp. 13-24.

Lillesand, T. et al. 2015. Remote Sensing and Image Interpretation. John Wiley \& Sons.

Perlin, K. 1985. An Image Synthesizer. In ACM Siggraph Computer Graphics 19.3, pp. 287-296.

Prensky, M. 2007. Digital Game-Based Learning. Paragon House, St. Paul.

Shute, V.J. and Zapata-Rivera, D 2012. Adaptive Educational Systems. Cambridge University Press, pp. 7 -27.

Streicher, A. et al . 2019. Usage Simulation and Testing with xAPI for Adaptive E-Learning. In: European Conference on Technology Enhanced Learning. Springer, pp. 692-695. 